\title{
La participación política de los inmigrantes en Italia: Un caso de participación mediada
}

\author{
Barbara D'Amen ${ }^{1}$ \\ Barbara.Damen@uniroma1.it \\ Massimo Lori ${ }^{2}$ \\ Malori@istat.it \\ Juan Manuel Pantoja Ypanaque ${ }^{3}$ \\ Manuel.Pantoja@uniroma1.it
}

Artículo de investigación científica y tecnológica recibido el 30/09/2010 y aprobado el 11/03/2011

\section{Resumen}

En el transcurso de las dos últimas décadas la presencia de extranjeros en Italia ha crecido considerablemente, dando lugar a relevantes consecuencias en el plano social, económico y demográfico.Sin embargo, las medidas de integración política puestas en acto, en particular aquellas previstas a nivel nacional, han resultado ineficaces, teniendo un impacto negativo en la vida de los ciudadanos inmigrantes, no logrando representar adecuadamente sus intereses de participación política y civil.

Paralelamente a la realidad política nacional, algunos municipios y gobiernos regionales han instituido, autónomamente, organismos muy significativos, como el Órgano de Consulta Elegida y la figura del Regidor Adjunto, en ausencia de un plano regulador nacional de referencia.

1 Bárbara D’Amen es doctoranda en Métodos de Investigación para el Análisis del Cambio Socio-Económico en el "Departamento de Análisis económico y social" (DAES) de la Facultad de Ciencias Políticas y Sociales de la Universidad de Roma "La Sapienza”. Sus intereses de investigación están dirigidos principalmente a la profundización de las diversas técnicas de análisis textual y al estudio de los fenómenos migratorios en una perspectiva metodológica enfocada en la interacción de técnicas cualitativas y cuantitativas.

2 Massimo Lori es investigador en el Instituto Nacional de Estadística Italiano, en el que se ocupa de las estadísticas de las instituciones sin fines de lucro. Ha publicado varios artículos sobre la participación social, el asociacionismo y las nuevas formas de activismo cívico.

3 Juan Manuel Pantoja Ypanaque es doctor en Métodos de Investigación para el Análisis del Cambio SocioEconómico en la Universidad de Roma "La Sapienza”, Italia. Colabora en el grupo de investigación “Corresponding Society on Contemporary Compulsory Labour” de la Universidad de Padua, en específico, indaga sobre el argumento de la inserción laboral y empresarial en la sociedad italiana de los migrantes latinoamericanos, con particular atención al caso peruano. 
Con el presente trabajo se han querido analizar de modo sistemático, en primer lugar, los instrumentos de representación política de los inmigrantes, implementados por las administraciones locales, con la finalidad de detectar, por una parte, algunos criterios que puedan resultar restrictivos o que limiten a los inmigrantes a participar plenamente en la vida política, y por otra parte,las experiencias de sucesos que pueden ser replicadas para reducir al mínimo tales impedimentos de participación.

Además, junto a las incipientes formas de participación convencional, han sido exploradas las formas de participación no convencional, nuevas formas de compromiso que han desarrollado los grupos de inmigrantes con la finalidad de evitar los obstáculos de participación, en particular, la difusión del asociacionismo entre los migrantes.

La idea de fondo, que emerge de este estudio, es que la actual estructura de oportunidades de participación política, por causa de la específica configuración de recursos y de orden institucional que ha asumido, aún no ha favorecido la movilización política de la población extranjera.En la práctica, las principales fuentes de legitimización de la participación se han manifestado mediante las organizaciones del tercer sector italianas y los sindicatos, que se han transformado en los canales privilegiados de la representación política de la población extranjera en Italia.

Palabras clave: Participación política migrante, asociacionismo migrante.

\begin{abstract}
During last two decades, the presence of people with other national identities in Italy has grown substantially affecting social,economic and demographic profiles. Measures of political integration, particularly those provided at the national level, have demonstrated ineffective, and failed to represent migrants' interests as far as political participation and civic life are concerned.

Similarly to the national political scenario and taking into account the absence of a national reference regulative framework, some municipalities and regions have independently established significant organisms like the elected councils or the representative of the Adviser (Consigliere aggiunto in Italian).

The aim of this paper is to analyze the institutions - formed by local governments - which assume political representation of migrants, identifying, on one hand, some criteria that may be restrictive or limiting immigrants' full participation in political life, and, on the other hand, the successful experiences that can be replicated to minimize these impediments to participation. Moreover, both conventional forms of participation and unconventional ones have studied, in addition to the new strategies that immigrant groups have developed in order to circumvent the barriers of participation such as the creation of Immigrants association. Finally, this paper shows that the current structure has not yet caused a strong political mobilization of the foreign population. The main sources of legitimation of immigrants participation in political life are Italian third-sector organizations and trade unions, which have become key channels for political representation of the foreign population in Italy.
\end{abstract}

Keywords: Political participation of immigrants, Immigrants association. 
1. La legislación italiana y el derecho de voto a los inmigrantes: entre Constitución y ley ordinaria.

$\mathrm{D}$ e acuerdo con el art. 48 de la Constitución italiana, el derecho de voto está reservado sólo a los ciudadanos italianos, excluyendo a los inmigrantes, que no han obtenido la ciudadanía, de la posibilidad de expresar sus opiniones políticas.Tal escenario con el tiempo,y con la intensificación de los flujos migratorios en Italia, ha conllevado a levantar numerosas controversias y propuestas, tanto de parte de la opinión pública como de las mismas fuerzas políticas. El debate en este sentido se ha desarrollado a través de dos trayectorias diferentes. Por un lado, están los llamados "garantistas", es decir,aquellos que de acuerdo con los principios de la Constitución proponen no variar los dictámenes constitucionales, sino solo una simplificación del trámite para la adquisición de la ciudadanía por parte de los extranjeros. Por otra parte, se encuentran los que proponen una revisión constitucional para establecer inequívocamente la plena legitimidad del voto de los extranjeros.

Una tercera alternativa es sostenida, en cambio, por aquellos que se basan en el art.10 de la Constitución, según el cual "la condición de los extranjeros está regulada por la ley en conformidad con las normas y tratados internacionales", sostienen que la reforma constitucional no es necesaria, sino que bastaría simplemente una intervención de los legisladores para establecer en modo preciso las condiciones bajo las cuales los extranjeros puedan ejercer sus derechos políticos. Esta última posición es apoyada también por las medidas elaboradas por la $\mathrm{UE}^{4}$, cuyo objetivo es promover la igualdad entre los ciudadanos nacionales y los procedentes de fuera de la UE. Emblemática al respecto es la "Convención sobre la participación de los extranjeros en la vida pública a nivel local" (1992) en favor de la concesión del derecho de voto a los residentes extranjeros, que Italia sólo ha ratificado en parte, manifestando una explícita reserva justo al capítulo C,dedicado a la ampliación del derecho de sufragio y elegibilidad para todos los residentes inmigrantes. Como es evidente de esta breve introducción existen muchas presiones, que vienen desde la esfera de la política comunitaria, para fomentar algún tipo de apertura de la legislación italiana, más tolerante hacia la participación de nacionales de terceros países. Italia, en este sentido, parece como si estuviera suspendida en una especie de estancamiento legislativo entre las demandas planteadas por la UE, inspiradas en el principio de derechos de los ciudadanos, y los dictámenes constitucionales basados en la explicitación y

4 El Consejo de Europa se ha manifestado explícitamente a favor de la concesión del derecho de voto a los extranjeros residentes con la "Convención sobre la participación de los extranjeros en la vida pública a nivel local" del 1992. La conclusiones de Strasburgo vienen sucesivamente reafirmadas por el Consejo Europeo de Tampere (1999) y por la "Resolución sobre la situación de los derechos fundamentales de la Union" (2001); en ambas se sostiene expresamente la necesidad de "acercar el estatus jurídico de los ciudadanos de terceros países al de los ciudadanos de los estados miembros” mediante la garantía de "una serie de derechos uniformes los más posible similares a aquellos de los cuales se benefician los ciudadanos de la UE” (Tratado de Tampere art.18-19). 
protección de los derechos de la ciudadanía. En este tipo de vacío legal se desencadena un sinnúmero de propuestas y experimentos que provienen de "abajo", es decir de las experiencias practicadas por las autoridades locales con poderes legislativos limitados, tales como los municipios, provincias y regiones. Muchas y variadas son las aplicaciones prácticas, que se dividen en dos modelos distintos: por una parte, el modelo del regidor extranjero adjunto $0^{5}$, y por otra, el ejemplo del órgano de consulta ${ }^{6}$.

\section{Regidor extranjero adjunto y la consulta de los migrantes}

En junio de 1994, cuando se realizaban las elecciones locales en el municipio de Nonantola en la provincia de Módena, se efectuó el nombramiento del primer Regidor Extranjero Adjunto. Esta nueva figura,completamente inédita en la escena política italiana, ya que se trata de una herramienta completamente inventada por las autoridades locales y no prevista por la legislación nacional, juega el importante papel de dar espacio y voz a los inmigrantes en el interior del organismo que mejor representa a la comunidad, es decir, el Concejo Municipal. En el caso específico del municipio de Nonantola, el mismo Consejo había determinado el número de dos Regidores adjuntos, en consideración a las diversas comunidades

5 En italiano el término exacto es: Consigliere straniero aggiunto. La palabra Consigliere, dependiendo de la terminología utilizada por los diversos países de habla hispana, puede ser traducida como Concejal; como en Ecuador, Consejero; en algunos municipios de Perú, y Regidor; en la mayoría de municipios de Perú y Colombia.

6 Que en las instituciones italianas es denominada en modo abreviado simplemente como La consulta. extranjeras presentes en dicho territorio. El Reglamento municipal preveía que los dos regidores adjuntos, elegidos directamente por los inmigrantes y con un mandato de igual duración que el Consejo Municipal, tuvieran derecho a:

- Asistir a todas las sesiones públicas del Consejo,

- Acceso a la zona para Regidores Municales,

- A ser informado de antemano y poder intervenir en el debate acerca de los asuntos en discusión, sin derecho de voto pero con la misma modalidad y tiempo establecido para los Regidores Municipales.

Las personas con derecho a votar, para elegir a los Regidores Municipales, fueron todos los extranjeros mayores de edad residentes en el municipio de Nonantola. La experiencia del Regidor Adjunto fue posteriormente emulada por muchas otras realidades en Italia, adoptándose en diversas formas, dependiendo de las necesidades expresadas por cada localidad, $y$ diferentes escalas (municipal, provincial y regional). Más allá de las variedades concretamente experimentadas (por ejemplo, los municipios más grandes como el de Ancona eligen solo a dos Regidores Extranjeros) las diferentes experiencias son enriquecidas también de elementos innovadores, así como positivos, que son atribuibles en primer lugar, a la elección para el encargo y, en segundo lugar, a la oportunidad de participar regularmente en el trabajo municipal, a diferencia de la Consulta, que no participa en las actividades del Concejo Municipal'.

7 En realidad también puede participar el presidente de la Consulta pero solo bajo expresa invitación del Consejo Municipal. 


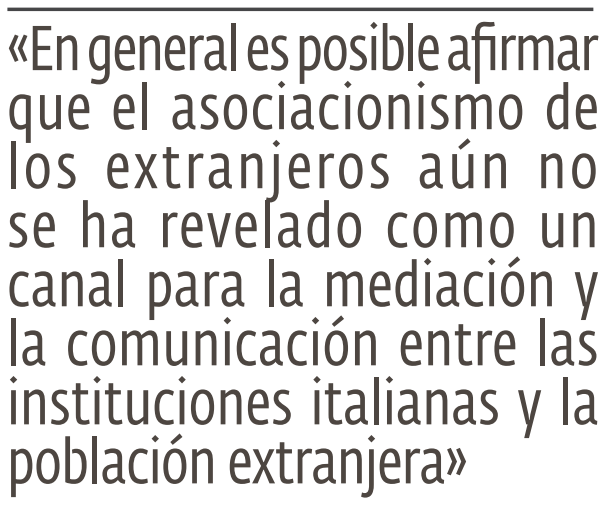

Por Consulta entendemos el organismo consultivo creado por las autoridades locales con el fin de facilitar la integración de los extranjeros. Una primera distinción entre las diferentes experiencias es sin duda aquella entre las Consultas Nombradas y las Consultas Elegidas.Las primeras, a diferencia de las segundas, prevén que la representación de los migrantes no se realice a través de la elección de los candidatos sino que se efectúe mediante nombramiento de parte de asociaciones de extranjeros reconocidas como representativas. En específico, en las Consultas Elegidas, se pueden verificar diferencias en relación a los mecanismos que regulan la consulta electoral, que pueden ser más o menos similares a aquellos reservados a los ciudadanos italianos, y vinculaban la base étnica o consideran la diferencia de género [Zincone 2005]. Por lo general, las consultas son mixtas puesto que prevén además de la presencia de los administradores locales y de los representantes de los extranjeros también de los representantes del mundo laboral, del tercer sector, de los sindicatos o de expertos de fenómenos migratorios.Las consultas se diferencian también en función del ámbi- to de la política en la que están llamadas a intervenir.En algunos casos, la consulta sólo tiene por objeto exclusivamente las cuestiones estrechamente relacionadas con el fenómeno migratorio, temáticas que de acuerdo a la normativa pueden ser definidas en modo más o menos detallado.En otros casos, sin embargo, el ámbito de competencia de la consulta va más allá de las cuestiones relacionadas con el discurso migratorio, refiriéndose a medidas de otra naturaleza.

En conjunto, ambos organismos descritos presentan fortalezas y debilidades. En cuanto respecta a la figura del Regidor Extranjero Adjunto, la posibilidad de participar en las reuniones municipales permite una socialización más rápida con la dinámica institucional y, por lo tanto, garantiza una mayor participación en el proceso de toma de decisiones políticas. Sin embargo, su función dentro de las reuniones municipales es sólo de asesoramiento o consulta, ya que no pueden ejercer su derecho al voto. Ventajoso en este sentido sería el nombramiento de un Regidor que sea a la vez presidente de la Consulta de los migrantes. ${ }^{8}$

En cuanto respecta al Regidor Adjunto, es necesario evidenciar que en la práctica resulta una figura aislada, respecto a organismos consultivos más amplios que pueden asumir sus actividades, y por lo tanto es poco representativo de las comunidades de inmigrantes. Por otra parte, aunque la elegibilidad sea un punto de fuerza, esta circunstancia de por

8 Esta solución ha sido efectivamente realizada por el municipio de Nonantola que en 1998 instituyó la primera "Consulta comunal elegida por ciudadanos extranjeros extra Unión Europea y apolides residentes en Nonantola" en el interior del cual se eligen los Regidores Extranjeros Adjuntos. 
sí no siempre es una garantía ante los problemas de representatividad, puesto que con frecuencia surgen problemas en la base electoral, que a menudo por cuestiones relacionadas a la diversa cultura de pertenencia, no se ve reconocida por los representantes elegidos. Este aislamiento además de limitar la función positiva y el aspecto incisivo de tal figura,implica una mayor exposición a los fenómenos de cooptación e instrumentalización política de los representantes de los inmigrantes desde lo institucional, favoreciendo la aparición de elementos de clientelismo en busca de futuros beneficios personales.

En general, en cuanto respecta a las consultas, éstas se han demostrado ineficaces órganos consultivos en la promoción de los derechos políticos y la participación de los migrantes. De hecho, lo que se evidencia de los resultados de investigaciones empíricas, la efectiva movilización de los migrantes ha encontrado dificultades importantes debido a que las consultas a menudo no han podido contar con fondos y disponibilidad de otros recursos que faciliten la participación de los extranjeros (por ejemplo,la entrega de dietas por la participación a las sesiones de consejo, la disponibilidad de sedes e instalaciones, etc.). Por otra parte, entre los representantes de los migrantes y la base electiva a menudo se ha verificado un déficit de representación, atribuible sobre todo a mutabilidad y opacidad de los procedimientos y proceso de selección candidatos.

En última instancia, ambos instrumentos ponen en acto un tipo de participación política mediada que, aunque reconocida a nivel institucional, carece de la capacidad para elaborar intervenciones incisivas y políticamente eficaces.
3. El cierre del sistema de los partidos políticos

Aunque no sean muchas las investigaciones que han analizado la participación de los inmigrantes en los partidos políticos, en general se evidencia un cuadro de sustancial refractariedad del sistema de partidos hacia los "nuevos ciudadanos". De hecho, un requisito previo a la inscripción, a menudo contemplado en los estatutos de los partidos (especialmente el de centro-derecha, como es el caso del principal partido político italiano,el PDL) es la posesión de la nacionalidad italiana. Por otra parte,la negación del derecho de voto, al menos a escala local, condiciona fuertemente una adecuada representación de los inmigrantes dentro de dichas estructuras. Es presumible, entonces, que la exclusión de la competencia electoral conlleve a un bajo peso e influencia de los inmigrantes dentro del partido político, donde parece prevalecer una lógica de cooptación o de instrumentalización antes que de representación real de las instancias de los inmigrantes [Carpo et al,2003].En modo transversal, parece ser generalizada, entre los partidos políticos, una desconfianza o renuencia a reconocer al inmigrado como sujeto político y no sólo como una persona que necesita asistencia.

Del análisis de los programas electorales de los partidos, sin embargo, emergen dos discursos políticos opuestos en el modo de conceptualizar el tema de la migración. Por un lado, los partidos de centro-derecha tienden a enfatizar el fenómeno en sentido negativo (la inmigración como problema) que debe ser tratado con políticas inspiradas en un modelo de inclusión de carácter tempo- 
ral o de asimilación [Ambrosini, 2005], con el objetivo de curar las situaciones de dificultad más agudas; por otro lado, los de centro-izquierda enfatizan en la presencia extranjera como un recurso y en la necesidad de adoptar políticas que amplíen los derechos de ciudadanía, valorizando la diversidad [Caponio,2006].

\section{Un sustituto de la participación política: el activismo en los sindicatos}

El sindicato cambia de piel. Con este tono evocador, se titulaba el artículo de un importante periódico italiano [Panorama, 2010] para describir la transformación que afrontan los sindicatos italianos, como resultado de los procesos migratorios. De hecho, en el transcurso de pocos años la representación de los extranjeros entre los inscritos a los sindicatos ${ }^{9}$ ha asumido una proporción bastante consistente, llegando a superar el 10\% del número total de miembros (sin tener predilección por algún sindicato en específico) e incluso por encima del $40 \%$ en algunas aéreas territoriales y categorías profesionales [Soldini, 2009; Mantovan, 2007; Caritas,2006; Carpo et al,2003; Mele, Enwereuzor, 2003]. Pero hay más. Dentro de las estructuras sindicales no es para nada raro que los extranjeros ocupen puestos de responsabilidad e importancia en calidad de representantes de empresas, ejecutivos nacionales o de sectores específicos [Zincone, 2000; Panorama, 2010; Carpo et al, 2003]. Además de las actividades institucionales, los migrantes inscritos a los sindicatos desarrollan la

9 Durante el 2008, los extranjeros inscritos a los cuatro principales sindicatos italianos, es decir CGIL, CISL, UIL y UGL eran respectivamente 300.000,332.561, 190.000 y 103.000 . doble función de mediadores culturales (entre el sindicato y los trabajadores extranjeros) y de sensibilización de los órganos sindicales en las cuestiones relacionadas con la inmigración [Leonardi, Mottura, 2002].

Las discretas tasas de inscripción, no son atribuibles simplemente al hecho de que ahora el sindicato es el único lugar donde los inmigrantes pueden elegir (votar) y sobre todo ser elegidos, sino a la centralidad que ya desde los años 80 las realidades sindicales han tenido en los procesos de integración de los extranjeros en la sociedad italiana. Tradicionalmente, el movimiento sindical italiano ha adoptado una política no corporativa y de apertura hacia los trabajadores, contrariamente a otras experiencias europeas, y esto como resultado tanto por la matriz solidaria de tipo católico-socialista,como por las características espontáneas y desorganizadas de la primera fase migratoria [Carpo et al, 2003]. De hecho, la acción sindical no se ha limitado a afrontar exclusivamente las temáticas laborales sino que se ha extendido a otros ámbitos [Mantovani,2007; Caritas,2006].Además de las cuestiones laborales, los sindicatos están llamados a intervenir en problemas y situaciones de dificultad que la población de inmigrantes enfrentan cotidianamente (la burocracia relacionada con los permisos de estadía, las relaciones con la policía ${ }^{10}$, concesión de préstamos, el reconocimiento de títulos de estudio, integración escolar de los niños, elección de la escuela, capacitación, búsqueda de empleo, problemas relacionados con la salud). En última instancia, la acción sindical se ha caracterizado en la lucha

10 En Italia es la policía la institución encargada de emitir los permisos de estadía. 
contra la discriminación institucional hacia los inmigrantes y en favor del reconocimiento de los derechos de la ciudadanía.

En esta dirección, sin embargo, la acción sindical encuentra limitaciones tanto de democracia interna, ya que los migrantes siguen estando sub representados en los órganos de decisión y en los puestos clave, como culturales, por ejemplo, la dificultad para enfrentar el tema de la inmigración sin separarla de las actividades institucionales rutinarias, considerándola como el epifenómeno de una transformación general del mercado de trabajo (inseguridad en el empleo y la desvalorización de la fuerza de trabajo) [Mantovan, 2007].

\section{Las asociaciones de inmigrantes}

En la actualidad, no es fácil describir el asociacionismo de los inmigrantes puesto que desde la investigación empírica se ha logrado sólo definir parcialmente algunos aspectos. La encuesta nacional en Italia, realizada en 2001, mostró que las asociaciones se encontraban localizadas principalmente en el centro-norte, es decir, en las regiones donde es mayor la presencia extranjera, con un campo de acción principalmente local (municipal o provincial) y mayoritariamente con una composición mono-étnica [Vicentini - Fava,2001].Otras investigaciones más específicas han identificado que los ámbitos priorizados por estas asociaciones son la integración en la sociedad de nueva residencia y la preservación y promoción de la cultura de origen. En la promoción de la integración, el asociacionismo organiza formas de ayuda-mutua para satisfacer las necesidades primarias (casa, trabajo, procesamiento de trámites burocráticos, apoyo financiero), y también aquellas necesidades relacionadas con el ámbito de la recreación y la socialización. Entre las actividades más estrechamente vinculadas a la esfera de la protección de la cultura de origen, se pueden considerar la organización de celebraciones y fiestas vinculadas a conmemoraciones políticas o religiosas del país de proveniencia y la enseñanza de la lengua materna a sus hijos, nacidos o criados en Italia.

La difusión de las asociaciones de inmigrantes se produjo en dos procesos diferentes: uno de abajo hacia arriba, como una exigencia de los extranjeros de auto-organizarse para hacer frente a necesidades de diferente tipo,y el otro de arriba hacia abajo, por efecto de un impulso externo (estado, instituciones públicas, las entidades religiosas, tercer sector) que han propiciado la aparición de un "asociacionismo inducido, que responde más a la mentalidad y las exigencias de la sociedad de nueva residencia que a las de los inmigrantes" [Schmidt di Friedberg, 1994,pp.151-152 ]. En efecto,la edad de oro del asociacionismo extranjero se puede situar aproximadamente entre años $80 \mathrm{y}$ 90, con la promulgación de la primera ley de inmigración (ley 943/1986,ley39/1990). Estas acciones legislativas, de hecho, ofrecían un nuevo espacio para la participación de los inmigrantes en la sociedad italiana, además de reconocer el derecho a la protección de la identidad cultural de origen [Noticias Iref, 1989, p. 9] y apoyar en el plano económico a las asociaciones [Caponio,2005].A partir de aquellos años, los inmigrantes han comenzado a unirse en asociaciones, de las cuales a menudo han sido reclutados, por las instituciones locales, los representantes de los extranje- 
ros.Además, para facilitar la participación de los inmigrantes, las organizaciones del tercer sector y las entidades religiosas han promovido la formación de nuevas asociaciones.

En general es posible afirmar que, el asociacionismo de los extranjeros aún no se ha revelado como un canal para la mediación y la comunicación entre las instituciones italianas y la población extranjera. Las asociaciones han presidido prevalentemente el ámbito de la cultura y del tiempo libre de la población extranjera, pero sin constituirse como un sujeto político y dotarse de una identidad colectiva fundamental para el surgimiento de un movimiento social o una movilización colectiva en condiciones de influir en la agenda política. La acción conjunta de los inmigrantes en los últimos años se ha centrado en "emergencias" particulares, como en el caso de las protestas para obtener el permiso de estadía en 2000 o en contra de la ley Bossi-Fini [Sciortino, 2003]. Por otra parte, el asociacionismo de los extranjeros se presenta discontinuo y frágil, altamente conflictivo internamente [Caselli y Grandi, 2009], carente de la estabilidad y la fiabilidad organizativa que le consentiría gozar de mayor legitimidad y poder dentro de la sociedad italiana.

\section{Conclusiones}

Es evidente, a partir de las experiencias descritas, que la participación política de los inmigrantes en Italia sigue siendo un fenómeno en evolución con vistas a conseguir legitimidad a nivel legislativo, pero sobre todo que garantice a los extranjeros la oportunidad de influir eficazmente en la elaboración de las políticas. Para llegar a ello se necesitan convergencias políticas hacia formas de participación que sean más flexibles e inclusivas. Esta posibilidad en cuanto respecta a la sociedad italiana parece aún remota.En los últimos años en efecto, después de la vitalidad de las propuestas y experiencias que han tenido lugar desde la década de los noventa, se ha presenciado una especie de estancamiento en lo que respecta a la posibilidad de elaborar nuevos y alternativos instrumentos de participación política en condiciones de superar la criticidad surgida de las experiencias consolidadas.Es así como la participación política de los inmigrantes en Italia se configura como un fenómeno de desarrollo problemático y discontinuo, incapaz de autoalimentarse con nuevas formulaciones y al mismo tiempo congelado por la inmovilidad política de la clase gobernante. La cual, incapaz de elaborar nuevas formas de participación política, más flexibles e inclusivas, se demora en la elaboración de propuestas legislativas diseñadas principalmente para contener y regular la entrada y permanencia legal en Italia ${ }^{11}$ sin tener en cuenta la voluntad

11 Emblemático en este sentido es la elaboración de parte del gobierno Berlusconi de un conjunto de leyes, conocidas como "Pacchetto sicurezza"; paquete de seguridad en español, aprobado por el gobierno en el mes de junio de 2009. Éste aporta una serie de novedades en la esfera del código penal, dicta una nueva disciplina en materia de adquisición de la ciudadanía e introduce el delito de clandestinidad.Además instituye la posibilidad para los alcaldes a organizarse en "rondas ciudadanas" con la finalidad de monitorear y contener fenómenos de posible desorden creados por los inmigrantes. Sin entrar en merito de un análisis exhaustivo del argumento es evidente que la clase dirigente in Italia tende a considerar la inmigracion como una amenaza de la cual defenderse y protegerse antes que una ocasión para experimentar una nueva forma de "hacer sociedad". 
de participación de todos los migrantes que viven ahora en modo estable en el territorio y que, mediante su trabajo contribuyen regularmente al crecimiento de la nación, tienen hambre de derechos reales y el deseo de hacer oír su voz.

\section{Bibliografía}

ABbATE C. «Il sindacato cambia pelle, Panorama", Visitado el 4 de enero del 2010, recuperado en la dirección: http://blog. panorama.it/italia/2010/01/04/il-sindacatocambia-pelle.

AMbrosini M. (2005) Sociologia delle migrazioni, Il Mulino, Bologna.

ASGI-FIERI (2005) «La partecipazione politica degli stranieri a livello locale", rapporto di ricerca commissionato dall'Assessorato alla Solidarietà sociale, Politiche giovanili e Programmazione sanitaria della Provincia di Torino, Descargable en la dirección: http://www.fieri.it/ktml2/files/uploads/attivita/papers\%20e\%20tesi/Rapporto_ASGIFIERI_2005.pdf.

CAPONIO T. (2005) Policy networks and immigrants' associations in Italy: the cases of Milan, Bologna and Naples, 'Journal of Ethnic and Migrations Studies', vol.31, n. 5, pp. 931-950.

CAPONIO T. (2006), «Quale partecipazione politica degli stranieri in Italia? Il caso delle consulte elettive dei comuni dell'Emilia Romagna", relazione presentata al IX Convegno internazionale della Società Italiana di Studi Elettorali (S.I:S.E.) La cittadinanza elettorale, Firenze, 14-15 dicembre 2006, descargable en la dirección: www.regione. toscana.it/elezioni/Documenti/IXConvegnoSISE/Caponio.pdf.

CAPONIO T. (2006a) Città italiane e immigrazione. Discorso pubblico e politiche a Milano, Bologna e Napoli, Il Mulino, Bologna.
CARITAS ITALIANA (2005) Immigrati e partecipazione politica. Dalle consulte e dai consiglieri aggiunti al diritto di voto, edizione Idos, Roma. CARPO F., CORTESE O., DI PERI R., MAGRIN G. (2003) «Immigrati e partecipazione politica. Il caso Italiano", rapporto di ricerca redatto nell'ambito del Progetto Satchel, Torino, descargable en la dirección: www. immigrazione.cefriel.it/download/dati/ partecipazione_straniera.pdf.

CASElli M., GRANDi F. (2010) "L'associazionismo dei migranti in Lombardia»,in Fondazione Ismu, 2010, pp.395-413. FONDAZIONE ISMU (2010) Dieci anni di immigrazione in Lombardia. Rapporto 2009, Franco Angeli, Milano.

LEONARDI S., MOTTURA G. (2002) Immigrazione e sindacato, Ediesse, Roma.

MANTOVAN C. (2006) Immigrazione e cittadinanza: autorganizzazione,partecipazione e rappresentanza degli immigrati in Veneto, in AIs (revisado por), Giovani sociologi, Civis, Napoli, pp 123-140.

MANTOVAN C. (2007) Immigrazione e cittadinanza: autorganizzazione, partecipazione e rappresentanza dei migranti in Italia, Franco Angeli, Milano.

MELI A.\& ENWEREUZOR U.C. «Participation of foreigners in public life at the local level", COSPE National focal point Italy,2003, descargable en la dirección: http://www.cospe. it/uploads/documenti/allegati/partecipazione_politica_dei_cittadini_stranieri_a_livello_locale.pdf.

NOTIZIE IREF (1989) Immigratie asociazionismo, n. 55/56, luglio-dicembre, pp. 5-32

Sciortino G.(2003) L'organizazione del proletariato immigrato in Italia, in Basso P.- Perocco F.(a cura di), Gli immigrati in Europa. Disuguaglianze, razzismo, lotte, Franco Angeli, Milano, 2003, pp.376-405 
SOLDINI P., "Servono più dirigenti immigrati”, 2009, visitado el 4 de Enero del 2010, ubicable en la dirección: http://www. stranieriinitalia.it/attualita-sindacati._soldini_cgil_servono_piu_dirigenti_immigrati_9615.html.

SPREAFICO A.(2006) Politiche di inserimento degli immigrati e crisi della banlieues. Una prospettiva comparata, Franco Angeli, Milano. VICENTINI A., FAVA T. (2001) Le associazioni di cittadini stranieri in Italia, ricerca promossa da CNEL, Analisi, Collana Ricerche n.37, Fondazione Corazzin, Venezia, www.fondazionecorazzin.it/web/article.asp?id=21.

Zincone G. (2000) Primo rapporto sull'integrazione degli immigrati in Italia, Il Mulino, Bologna. 
